\title{
Dichroism of Poly(Methyl Methacrylate) Thin Films Doped with Disperse Orange 11 Molecules
}

\author{
B. AbBas* And M. Alshikh Khalil \\ Atomic Energy Commission, P.O. Box 6091, Damascus, Syria
}

(Received November 18, 2008; in final form April 21, 2009)

\begin{abstract}
We investigate the absorption spectra of poly (methyl methacrylate) (PMMA) thin films doped with Disperse Orange 11 (DO11) dye molecules which change under illumination with visible light. Dichroism measurements of the kinetics of the transmission changes at $472 \mathrm{~nm}$ at room and low temperatures $\left(-60^{\circ} \mathrm{C} \pm 2^{\circ} \mathrm{C}\right)$ have been investigated. Our data show that the photoisomerisation reaction and the light-induced polar orientation depend on the molecular structure of the polymer. Simultaneous UV-visible spectroscopy at low temperature confirms the existence of two forms of DO11 molecules (keto and enol forms), which are responsible for the interaction with irradiating laser light and forming an anisotropic structure inside the PMMA/DO11 thin film.
\end{abstract}

PACS numbers: 33.55.+b, 78.20.e, 78.66.Qn

\section{Introduction}

The importance of organic and polymeric nonlinear optical (NLO) materials for applications in nonlinear optics is widely recognised, and there has been intensive research on these materials in the past decade $[1,2]$. Recently, photoinduced optical anisotropy in polymer/organic dye systems has been the subject of intensive investigations $[3,4]$. These materials are promising in the field of modern optics for optical recording, optically-controlled optical elements, optical switches, and so on. Numerous studies on azo dyes incorporated in polymeric systems have been reported in the past two decades [3-5]. Anthraquinone dyes have also been under extensive research as well, since they have interesting optical properties. Of special interest, nonlinear optical properties of DO11 dye have been explored in the field of solid dye lasers owing to its recognised optically pumped lasing action at the attractive wavelength of $650 \mathrm{~nm}$. The dye was incorporated into PMMA rods, and amplified spontaneous emission was studied under second-harmonic Nd:YAG laser excitation in a transverse pumping configuration. Gain and conversion efficiency were found to be comparable with those for other laser dyes $[6,7]$. Measurements of the absorption of linearly polarised light by an ensemble of oriented molecules form also a very important method available for studying the structure and physical behaviour of nonlinear optical guest-host systems. In other words, dichroism is a measure of the orientation of the nonlinear optical dye molecules in the host polymer [8].

In this paper, based on the traditional pump-probe technique, the linear dichroic properties of PMMA/DO11

* corresponding author; e-mail: scientific@aec.org.sy (B. Abbas) guest-host polymeric thin films were studied. Continuous argon laser light was used to pump and probe the samples. In addition, UV-visible spectrometry was used as a spectroscopic tool for simultaneous characterisation at room and low temperatures, so that the photoisomerisation and polar alignment of DO11 molecules are presented.

\section{Experimental}

\subsection{Sample preparation}

Poly(methyl methacrylate) (PMMA, MW $=36000)$ from Acros Organics, and 1-amino-2methylanthraquinone (Disperse Orange 11, DO11, $\approx 95 \%$ dye content) from Aldrich are the starting materials for preparing the guest-host polymeric samples. The film samples were prepared as follows: PMMA (2 g) was dissolved in $20 \mathrm{ml}$ of dichloromethane $\left(\mathrm{CH}_{2} \mathrm{Cl}_{2}\right)$. A clear solution was obtained. DO11 (equivalent to $5 \%$ of PMMA by weight) was added to this clear solution. The mixture was then stirred for 5-6 h until a clear, orange solution was obtained. Thin films with thicknesses (measured by the prism coupling technique) of the order of $1-2 \mu \mathrm{m}$ were dip-coated on glass substrates. Directly following the dip-coating procedure, samples were baked in an oven and held at $70^{\circ} \mathrm{C}$ for one hour in order to eliminate the residual solvent.

\subsection{Experimental setup}

Upon pumping of the dye molecules with linearly polarised light at $472 \mathrm{~nm}$ the guest-host polymeric thin films become dichroic. The absorbances parallel $\left(A_{\|}\right)$and perpendicular $\left(A_{\perp}\right)$ to the electric vector of the pump beam become different. However, the dichroism could be measured using another laser beam linearly polarised 
(probe) to let it pass through the sample and then measure the absorbance of this light when it is polarised both parallel and perpendicular to the polarisation direction of the pump beam. However, it is very important to use a very weak probe beam with a wavelength very close to the absorption maximum of the dye molecules where any changes in the absorption will be greatest. In order to find the best suitable absorption wavelength for the samples, a UV-visible spectrophotometer (Photodiode Array Photospectrometer (PDA) Specord S100, from Analytik Jena) was used to record the UV-visible absorption spectrum. Figure 1 shows that the maximum absorption wavelength takes place at $470 \mathrm{~nm}$. Hence, a light with a wavelength of $472 \mathrm{~nm}$ provided by the Ar-ion laser was used for both pump and probe beams. It is worthwhile to mention here that the reference sample of PMMA film alone on the substrate was measured in the beginning; and then all subsequent UV-visible spectroscopic measurements were normalised to it. This procedure assures the removal of the effect of the polymer and the substrate absorption on later measurements. Photoinduced dichroism was measured using the experimental arrangement shown in Fig. 2.

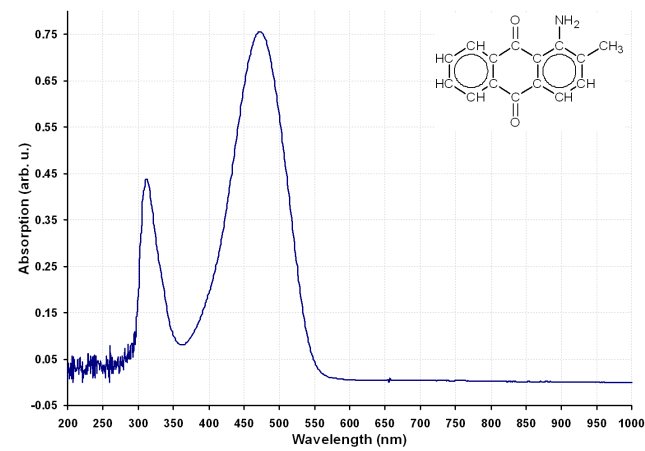

Fig. 1. UV-visible spectrum of PMMA thin film doped with DO11 (5 wt\%).

Argon-ion laser (543-MAP-A02, from Melles Griot) was used to generate linearly polarised light (472 nm) as the pump beam to induce the dichroism in the sample. The probe beam was generated using a microscope glass slide as a beam splitter placed in the way of the pump beam. The probe beam was made as weak as possible by means of some neutral density filters and let to pass through the sample, so that the probe beam did not contribute to the induced dichroism in the sample by the pump beam. The half-wave plate $(\approx 488 \mathrm{~nm})$ was used in the probe beam path in order to alter its polarisation state from parallel-to-the-pump-beam polarisation to perpendicular to it and vice versa so that both $\left(A_{\|}\right)$ and $\left(A_{\perp}\right)$ could be measured. An additional polariser was used in the probe beam path to ensure the correct polarisation after the probe beam passes through the half-wave plate. The intensity of the probe beam after passing through the sample was measured using a photodiode and read by a computer through a low noise current

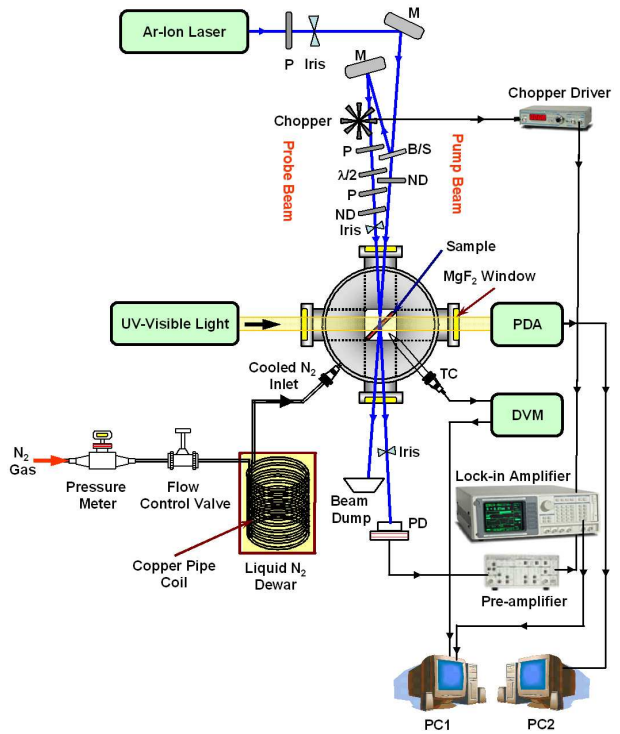

Fig. 2. Apparatus for measuring dichroism in dye-doped polymer films at room and low temperatures ( $\mathrm{P}$ - polarizer, $\mathrm{PD}$ - photodiode, ND - neutral density filter, $\mathrm{M}$ - aluminium mirror, BS - beam splitter, TC - thermocouple, $\lambda / 2$ - half-wave plate, DVM $6_{1 / 2}$ Digit Multimeter, PDA - photodiode array).

preamplifier (SR570, from Stanford Research Systems), and a DSP lock-in amplifier (SR850, from Stanford Research Systems). An IEEE 488.2 GPIB (from National Instruments) card was used to control and record the experimental data along with a special program written in Borland $\mathrm{C}++$, so that a plot of the transmitted probe light intensity versus time is generated. The absorbances were then calculated from

$$
A_{\|}=-\log _{10}\left(\frac{I_{\|}}{I_{0}}\right)
$$

and

$$
A_{\perp}=-\log _{10}\left(\frac{I_{\perp}}{I_{0}}\right),
$$

where $I_{0}$ is the intensity of the probe beam reaching the detector when there is no sample, and $I_{\|}$and $I_{\perp}$ are the intensities of the probe beam transmitted through the sample when it is polarised parallel and perpendicular to the pump beam polarisation, respectively.

\section{Results}

\subsection{Irradiation at room temperature}

Figure 3 shows a representative experimental result for a $1.5 \mu \mathrm{m}$ thin film. The pump beam intensity was $\approx 16 \mathrm{~mW} / \mathrm{cm}^{2}$ with vertical polarisation, while the probe beam intensity was $\approx 0.03 \mathrm{~mW} / \mathrm{cm}^{2}$ for both horizontal and vertical polarisations. The plot labelled $I_{\|}$is for the case of both the pump and probe beams are parallel in the $s$-polarisation state while the plot labelled $I_{\perp}$ is for the case of which the pump beam has an $s$-(vertical) polarisation state and the probe beam has a $p$-(horizontal) 


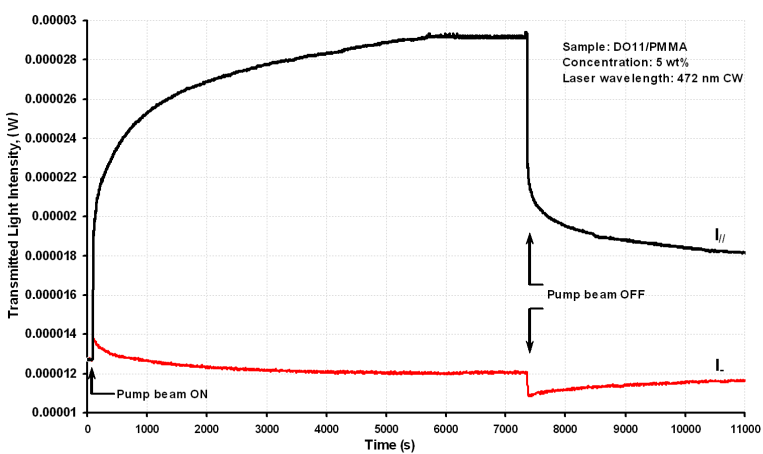

Fig. 3. Photoinduced dichroism in a PMMA/DO11 (5 wt\%) film $(\approx 1.5 \mu \mathrm{m})$. Pump light intensity was $\approx 16 \mathrm{~mW} / \mathrm{cm}^{2}$, and the probe light intensity was $\approx 0.03 \mathrm{~mW} / \mathrm{cm}^{2}$ (both beams are at $472 \mathrm{~nm}$ ).

polarisation state. In the case of both probe and pump beams are of the same polarisation state, a fast increase in the transmitted light intensity was recorded as soon as the pumping started. This fast increase in the transmitted light intensity was then, followed by a slower increase, until an equilibrium state was reached.

It can be seen that switching off the pumping source led to a fast decay of the transmitted light intensity in the beginning, which was followed by a slower decrease. This slow decay took very long time in order to reach a stationary state, in which no further decrease was detected. It is noticeable that the transmitted light intensity was detected long after switching the pumping sources off, and did not decay completely to the original level before conducting the experiment. In the case of using the probe and pump beams at orthogonal states of polarisation, the transmitted light intensity increased slightly for a short time. Then, it started to decrease slowly and reached an equilibrium state, at which the transmitted light intensity had a constant level, which was slightly lower than the initial level before the beginning of the experiment. Switching off the pump light causes the transmitted light intensity to decrease further below the initial level. However, the level of the transmitted light intensity starts to recover slowly, and reaches the initial level after a long time.

\subsection{Irradiation at low temperature}

Earlier studies on dilute liquid samples of PMMA/DO11 showed that under basic condition, DO11 undergoes a structural rearrangement in which the inter- or intramolecular transfer of hydrogen (a proton) in photoexcited DO11 molecules takes place. This is due to the newly formed charge density distribution of the photoexcited DO11 molecule which favours the transfer of a proton. The transferred proton normally binds to an oxygen atom yielding an $\mathrm{OH}^{-}$group [9]. Based on the DO11 molecular structure shown in Fig. 4, it seems reasonable that a hydrogen could readily bind to one of the available lone oxygens. This form is more chemically stable than the molecules without hydrogen bonds (keto type). Upon the absorption of light energy, the dye structure changes from a stable keto form in the ground state to a less stable enol form in the excited state. This enol form will mostly give off its absorbed energy rapidly and return to the keto form [9]. This process is referred to as phototautomerisation process, in which an intramolecular proton transfer takes place as a result of photoirradiation [10, 11]. Figure 4 shows the phototautomerisation process reaction [9]. Furthermore, PMMA matrix can be considered as a Lewis base $[12,13]$, so that the keto form of DO11 is likely to be very much present in the guest-host polymeric system.

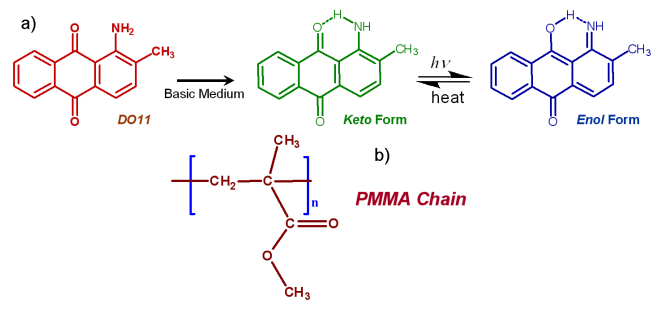

Fig. 4. (a) Phototautomerisation reaction under basic condition and light pumping. (b) The chemical structure of PMMA.

Figure 3 shows that during the pumping process, an equilibrium state is reached, in which the system contains a constant fraction of each form. In this photostationary state, conversion between both forms is continually taking place in both directions, but the photochemical keto $\Rightarrow$ enol rate is equal to the photochemical plus the thermal enol $\Rightarrow$ keto rates. There are a few ways to reduce the $e n o l \Rightarrow k e t o$ decay rate. One of them is to reduce the thermal decay via reducing the sample temperature to the value that no ambient heat will affect the enol form and force it to convert to the keto form. Alternatively, if during irradiation a photostationary state is formed, a fraction of the DO11 molecules exists in the enol state. To investigate these conditions, two techniques have been followed, the first one is to conduct the dichroism experiments at sub zero ${ }^{\circ} \mathrm{C}$, and the second approach is to pump the cooled sample with linearly polarised laser light and monitor the change in the UV-visible absorption spectra during irradiation.

To take the advantage of both approaches, an innovative experimental technique was adopted $[14,15]$. In this technique, dichroism measurements were conducted and, at the same time, UV-visible spectra were recorded at interval times (300 s intervals) during the experiments; all under the control of the sample temperature. A special holder was used to hold the samples firmly in the centre of the sample chamber. The special holder sits with the sample in an aluminium block, which contains copper pipes to let the nitrogen gas (oxygen free) pass through. The coolant nitrogen gas was cooled in a copper coil immersed in liquid nitrogen. Also, the temperature was monitored by a $T$-type thermocouple, and controlled 


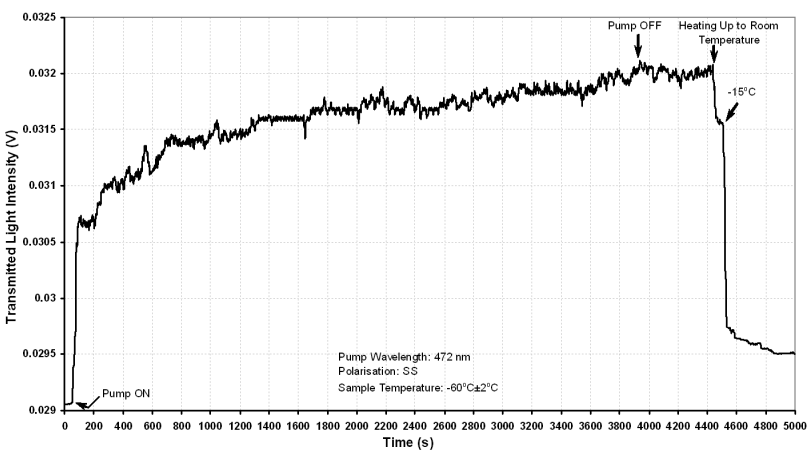

Fig. 5. Photoinduced dichroism in a PMMA/DO11 (5 wt\%) film $(\approx 1.5 \mu \mathrm{m})$. Pump light intensity was $\approx 16 \mathrm{~mW} / \mathrm{cm}^{2}$, and the probe light intensity was $\approx 0.03 \mathrm{~mW} / \mathrm{cm}^{2}$ at $-60^{\circ} \mathrm{C}$ (polarisation states of pump and probe beams are vertical).

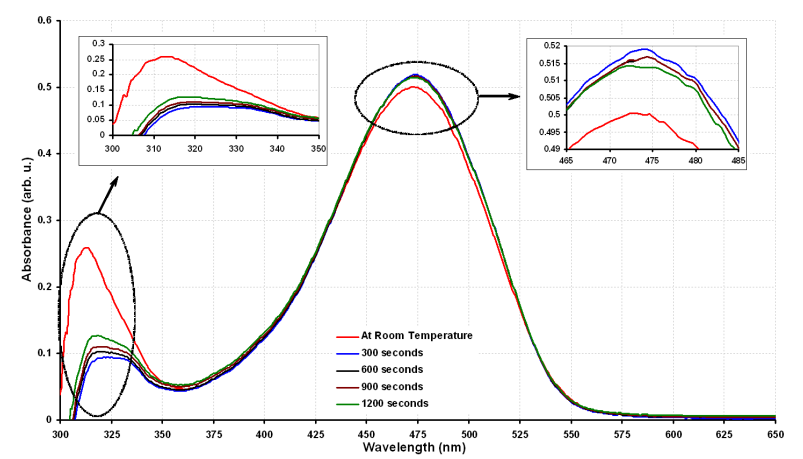

Fig. 6. Absorption changes of a sample of PMMA/DSR1 thin film (5 wt\%) pumped by laser light. Intensity was $\approx 16 \mathrm{~mW} / \mathrm{cm}^{2}$, and the probe light intensity was $\approx 0.03 \mathrm{~mW} / \mathrm{cm}^{2}$. (Polarisation states of pump and probe beams are vertical.) The sample temperature was kept at $60^{\circ} \mathrm{C}$. The left inset shows the absorption increase in the range 300-350 nm during irradiation, while he right inset shows the absorption decrease in the range $450-550 \mathrm{~nm}$ during irradiation.

by adjusting the flow rate of the nitrogen gas through a valve. The thermocouple consisted of two arms, one of which is immersed in liquid nitrogen (reference arm), and the second of which is inserted through a tiny hole on the top of the sample chamber and fixed very close to the sample (sample arm). This thermocouple was connected to a very sensitive high resolution digital voltmeter ( $6_{1 / 2}$ Digit Multimeter, Model: 2000, from Keithley) so that the voltage difference between the two arms of the thermocouple is digitally measured. The output of the digital voltmeter was connected to the GPIB card and a suitable mathematical conversion routine was used to convert these readouts into temperature degrees. The temperature was shown on the controlling panel of the software (on the PC screen) and, thus, fast modification to the sample temperature was carried out immediately as needed via the flow valve. Temperature readout was recorded at every point during the optical measurements.
With this setup, the temperature can be very much reduced down to $180^{\circ} \mathrm{C}$ (with fluctuations of $\pm 1^{\circ} \mathrm{C}$ above $0^{\circ} \mathrm{C}$, and $\pm 2^{\circ} \mathrm{C}$ below this), and the spectrum can be measured simultaneously as the light pumping is taking place because of different optical paths that pump and UV-visible-probe beams follow. After passing through the sample, the collimated UV-visible-probe beam (first arm of light) is delivered to the entrance of the photodiode array system of the spectrometer. On the other hand, both pump and probe laser beams from Ar-ion laser (second arm of light) entered the sample chamber following an orthogonal path to the UV-visible-probe beam. Needless to say that all beams hit the polymeric sample at the same point (the sample is at 45 degrees to each arm of the beams), so that the optical dichroic properties and the corresponding change in the absorption spectra are measured simultaneously at the desired temperature. Thus, a PMMA/DO11 sample was placed in the spectrometer compartment (inside the cooling chamber) and, using cooled $\mathrm{N}_{2}$ gas, the sample temperature was kept at $-60^{\circ} \mathrm{C} \pm 2^{\circ} \mathrm{C}$. Spectra were taken at time intervals of $300 \mathrm{~s}$. Figures 5 and 6 show the experimental result. The final spectrum shown in Fig. 6 was stable at $-60^{\circ} \mathrm{C}$ for at least one hour. The original spectrum $(100 \%$ keto) was substantially recovered by subsequent heating the sample to room temperature. A discussion of these results is laid out in the following section.

\section{Discussion}

Photoinduced reorientation of DO11 dye molecules depends on host polymer structure and dye concentration. In order to explain the plots in Figs. 3 and 5, the molecular distribution of the dye molecules within the polymeric matrix has to be considered. If the angular distribution of the keto form is biased in a particular direction, then the material can be considered as anisotropic. This means that the absorption characteristics of this material are also angularly dependent [16]. However, the molecular anisotropy of the dye molecules plays an important role as well, in which the anisotropy of the keto forms can lead to a bias in their angular distribution. This occurs during pumping with polarised light so that the probability for a keto molecule to be pumped into the enol state upon irradiation is proportional to the angle between the long axis of the keto molecule and the beam polarisation direction [15]. Looking at Fig. 7, the orientation of the keto form are defined using polar coordination by the angles $\theta$ and $\varphi$. The probability, $P$, for a keto form molecules to be pumped into the enol state is then given by

$$
P \propto \cos ^{2}(\theta) \sin ^{2}(\varphi) .
$$

The absorption probability, proportional to $\cos ^{2} \theta$, leads to a selective depletion of the keto molecules parallel to the pump light polarisation direction and resulting in having more enol molecules. This generates a "hole" in the angular distribution of the keto molecules along the pump beam polarisation direction [17]. Molecules lying in the $x$-direction will be pumped preferentially to 


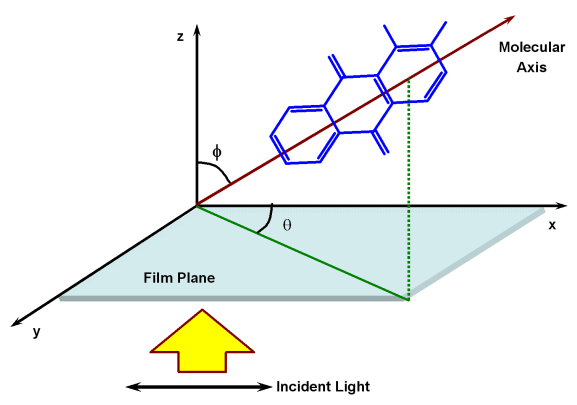

Fig. 7. Dye molecule orientation with respect to the polarisation direction of the incident light.

the enol state. This leads to having the keto population biased towards the $y$-direction. Enol molecules have weaker absorption, so that a probe beam polarised in the $x$-direction ( $A_{x}$ or $A_{\|}$, parallel to that of the pump beam) does not suffer from absorption as it travels through this sample. Moreover, a probe beam polarised in the $y$-direction ( $A_{y}$ or $A_{\perp}$, perpendicular to the direction of that of the pump beam) will experience a little reduction in absorption. It is important to notice that angular hole burning leads to a decrease in the absorption in all directions for wavelengths close to the absorption maximum of keto forms. Pump light intensity, and the lifetime of the enol form plays an important role in the extension and the effectiveness of the angular hole burning. For example, if the lifetime of the enol form is very long or the pump intensity is very high, then eventually most keto forms would be pumped into the enol state.

Another model has also to be considered, which is called angular redistribution (AR) model. This model was widely used by Russian researchers [17] to describe how films become birefringent upon irradiation with light. In this model, the relaxation time is very short so that there is no depletion in the population of keto molecules. In other words, the keto molecules are selectively pumped into the enol state according to the $\cos ^{2} \theta$ angular dependence of Eq. (3) and after a very short time they relax back to the keto state either thermally or through photoexcitation with a new orientation [18]. However, if the final keto molecule, after the photoisomerisation cycle, still lies along the polarisation direction of the pump light, it will go through a keto-enol-keto process again. On the other hand, if this keto molecule has another orientation, it is less likely to go through this process. As a result of this continuous angular selective interaction, more keto molecules are gradually aligned in a plane perpendicular to the polarisation direction of the pump light. This causes the absorption $\left(A_{\|}\right)$parallel to the polarisation direction of the pump light to decrease due to molecules being pumped away from this direction. On the other hand, the absorption $\left(A_{\perp}\right)$ perpendicular to the polarisation direction of the pump light increases as a consequence of more molecules being aligned in this direction. Although the number of molecules parallel to the pump polarisation direction decreases while the number of molecules perpendicular increases, the total number of keto molecules is constant, one obtains [16] $\Delta A_{\|}=-2 \Delta A_{\perp}$. In other words, the depletion of the keto molecules away from the polarisation direction of the pump beam does not go to completion because of the thermal energy of the keto molecules that causes random motion leading to oppose the alignment process [15]. Switching the pump beam off leads to have the anisotropy relaxing due to angular diffusion of the molecules [16].

Looking back at Fig. 3 one can see that during the signal rising right after switching the pump light on, two distinctive parts can be distinguished. The first part is a fast rise in the transmitted light. This can be explained according to the angular hole burning (AHB) model as the keto molecules are being depleted selectively and this process is dominant. In the second part, as the angular redistribution takes the advantage, the transmission of the perpendicular component decreases progressively. Switching the pump light off causes both transmissions to decrease suddenly due to fast relaxation of the enol form; but the dichroism originating in the molecular orientation has much larger relaxation time.

The absorbance changes in the sample in the case of using both polarisation states of the probe beam (parallel and perpendicular to the polarisation direction of the pump beam) can be calculated from Eqs. (1) and (2). The result of such calculations is shown in Fig. 8. In the

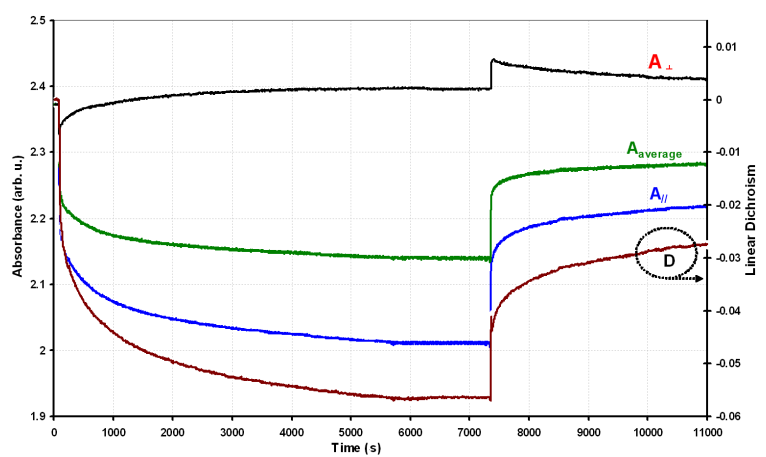

Fig. 8. Photoinduced absorption and dichroism generated in a PMMA/DO11 (5 wt\%) film whose thickness is $\approx 1.5 \mu \mathrm{m}$. The sample was pumped by linearly polarised (472 $\mathrm{nm})$ light, and probed by (a) p-polarised light $\left(A_{\perp}\right)$, and $(\mathrm{b}) s$-polarised light $\left(A_{\|}\right)$at $472 \mathrm{~nm}$, respectively.

case of using probe and pump beams with orthogonal polarisation states, the sample absorption decreases by a small amount as soon as the pump beam is switched on. However, a small increase in this absorption follows this stage in a very slow manner until a stationary state is reached so that there is no further increase in the absorption. Nevertheless, switching off the pump beam leads to further increase in the sample absorption, and the overall change is rather small. In the other case where the 
probe and pump beams polarisation states are similar to each other, the sample absorption decreases substantially upon switching on the pump beam. This decrease is fast in the beginning, followed by a slower rate of increase until a stationary state is reached upon which no further decrease in absorption is recorded. Cutting off the pump beam leads to a fast increase in the absorption in the beginning followed by a slower increase, which takes longer time to reach a stable value. The kinetics of keto $\Rightarrow$ enol conversion can be studied depending on information obtained from absorption spectra $A_{\|}$and $A_{\perp}$ obtained from dichroism experiments. This can be done by looking at the average absorbance, $A_{\mathrm{a}}$, which is given by [3, 19],

$$
A_{\mathrm{a}}=\frac{A_{\|}+2 A_{\perp}}{3} .
$$

This average absorbance represents the isotropic absorption. In other words, it does not depend on the medium anisotropy, and it characterises only the relative concentration of keto forms of DO11 molecules present in the sample. Figure 8 represents the calculated $\left(A_{\mathrm{a}}\right)$ values according to Eq. (4). This figure shows clearly how the concentration of keto form changes with analogy to AHB and $\mathrm{AR}$ processes. In the beginning, this decrease in the keto population takes place in a fast fashion indicating a fast growth in the population of the enol form as long as the AHB is the dominating process. However, as the AR process starts to dominate, the decrease in the keto population starts to slow down as an indication of slowly reaching a photostationary state. At this photostationary state, the concentration of both forms tends to be constant, in which the overall number of keto molecules converting to the enol form is equal to that of the enol forms converting to the keto form. This photostationary state manifests itself in Fig. 8 as a plateau in $A_{\text {a }}$ values. The dichroic ratio (the order parameter) was computed from the recorded spectra as [19]:

$$
D=\frac{A_{\|}-A_{\perp}}{A_{\|}+2 A_{\perp}} .
$$

The kinetics of the calculated dichroism emphasises the ability for DO11 molecules to react with the pump laser light and form a polar order in the sample in a fast fashion. This value reaches a plateau in analogy with the photostationary states of the polymeric system. However, it also diminishes as soon as the pump irradiation is cut off, manifesting the tendency of the polymeric system towards the isotropic state of the DO11 molecule distribution within the polymer network. These observations indicate that these changes are completely reversible, leading to the conclusion that keto $\Rightarrow$ enol of DO11 was occurring in the guest-host polymeric thin film. The observed characteristic changes of the absorption spectrum, a reduction in the main absorption peak and an increase in the absorption at lower wavelengths as well as the appearance of an isosbestic point in the range of 400-425 nm, are typical of experiments carried out on this polymeric system. Figures 5 and 6 illustrate the absorption characteristics of PMMA/DO11 thin films under cold conditions.

Looking at Fig. 3 and Fig. 5 one can see that there is a slightly different behaviour between them. Namely, in Fig. 5, there is a slow relaxation in the recorded light signal between temperatures $-60^{\circ} \mathrm{C}$ and $-15^{\circ} \mathrm{C}$, followed by a fast relaxation behaviour in a similar fashion to that in Fig. 3. This might be attributed to lack of thermal energy necessary for DO11 molecules to decay into an isotropic distribution within the polymer network. Another difference is noticed as well as the pump beam is switched off. The level of generated anisotropy in the sample does not change as long as the sample temperature is held stable at $-60^{\circ} \mathrm{C}$, which can be attributed to reduced thermal relaxation at this temperature. Figure 6 shows the simultaneous absorption spectra of the sample that was under the dichroic investigation appearing in Fig. 5. The first note in this figure is that the absorption spectrum at room temperature is different from that at low temperature. It is clear that absorption band in the range $450-550 \mathrm{~nm}$ is lower than that at low temperature, as well as the absorption band in the range 300-350 nm is higher than that at low temperature. Nevertheless, as the dichroism investigation proceeds, a reduction in the main absorption peak and an increase in the absorption at lower wavelengths are clearly evident in the insets of Fig. 6. The stability of the final spectrum following irradiation for a long time at $-60^{\circ} \mathrm{C}$ indicates that at this temperature, the thermal enol $\Rightarrow$ keto decay rate was very small. Therefore, the final spectrum corresponds to a photostationary state at which the competing photochemical reactions were taking place at equal rates. Moreover, the fact that the absorption at $320 \mathrm{~nm}$ increased by $\approx 15 \%$ indicates that the enol form has significant absorption at this wavelength. However, this does not mean that the recorded final spectrum corresponds to $100 \%$ of enol forms, simply because such large enol absorption at $320 \mathrm{~nm}$ would lead to considerable pumping back to the keto state. In other words, the final spectrum represents a mixture of both enol and keto forms.

\section{Conclusion}

In this study, the absorption characteristics of PMMA thin films doped with the DO11 dye molecules under illumination with visible light were investigated. Dichroism measurements of the kinetics of the transmission changes at $472 \mathrm{~nm}$ at room and low temperatures have also been studied. It was shown that the phototautomerisation reaction is dependent on the chemical structure of the polymer as well as the photoactive dye. Simultaneous UV-visible spectroscopy at low temperature showed that there exist two forms of DO11 molecules called keto and enol forms. Keto and enol forms are responsible for the interaction with pumping laser light and forming an anisotropic structure inside the PMMA/DO11 thin film. However, all photoinduced changes in UV-visible absorption and dye alignment are reversible. PMMA/DO11 guest-host polymeric systems tend to relax to their stable thermodynamic state as soon as the 
pump light is removed and their temperature is close to room temperature.

\section{Acknowledgments}

The authors would like to express their thanks to the Director General of AECS Prof. I. Othman for his continuous encouragement, guidance and support.

\section{References}

[1] Z. Sekkat, M. Dumont, Appl. Phys. B 54, 486 (1992).

[2] M. Ivanov, L. Nikolova, T. Todorov, N. Tomova, V. Dragostinova, Opt. Quantum Electron. 26, 1013 (1994).

[3] Z. Sekkat, P. Prêtre, A. Knoesen, W. Volksen, V.Y. Lee, R.D. Miller, J. Wood, W. Knoll, J. Opt. Soc. Am. B 15, 401 (1998).

[4] I.G. Marino, R. Raschell, P.P. Lottici, D. Bersani, C. Razzetti, A. Lorenzi, A. Montenero, J. Sol-Gel Sci. Technol. 37, 201 (2006).

[5] C. Chun, E. Seo, M.J. Kim, Y.D. Shin, J.S. Lee, D.Y. Kim, Opt. Mater. 29, 970 (2007).

[6] B.F. Howell, M.G. Kuzyk, J. Opt. Soc. Am. B 19, 1790 (2002)

[7] B.F. Howell, M.G. Kuzyk, Appl. Phys. Lett. 85, 1901 (2004)
[8] K. Yamaoka, E. Charney, J. Am. Chem. Soc. 94, 8963 (1972)

[9] W. Chu, S.M. Tsui, J. Environ. Eng., 127741 (2001).

[10] T. Kobay, T. Kobayashi, in: Photoreactive Organic Thin Films, Ed. Z. Sekkat, W. Knoll, Academic Press, San Diego 2002, p. 49.

[11] S.A. Ahmed, A.A. Abdel-Wahab, H. Dürr, in: Handbook of Organic Photochemistry and Photobiology, 2nd ed., Eds. W. Horspool, F. Lenci, CRC Press, Boca Raton 2003, p. 96-11.

[12] F.M. Fowkes, D.O. Tischler, J.A. Wolfe, L.A. Lannigan, C.M. Ademu-John, M.J. Halliwell, J. Polym. Sci., Chem. Ed. 22, 547 (1984).

[13] N. Shahidzadeh, M.M. Chehimi, F. Arefi-Khonsari, J. Amouroux, M. Delamar, Plasmas Polymers 1, 27 (1996).

[14] N.R. King, E.A. Whale, F.J. Davis, A. Gilbert, G.R. Mitchell, J. Mater. Chem. 7, 625 (1997).

[15] B. Abbas, Ph.D. Thesis, The University of Reading, Reading UK 1999, p. 100.

[16] Z. Sekkat, M. Dumont, Synth. Met. 54, 373 (1993).

[17] M. Dumont, Z. Sekkat, SPIE Proc. 1774-24, 188 (1992).

[18] T. Todorov, L. Nikolova, N. Tomova, Appl. Opt. 31, 397 (1984).

[19] T. Watanabe, J.L. White, Colloid Polym Sci. 270 , 519 (1992). 